\title{
Chromatin profiling of cortical neurons identifies individual epigenetic signatures in schizophrenia
}

\author{
Fedor E. Gusev1,2, Denis A. Reshetov², Amanda C. Mitchelli, Tatiana V. Andreeva 2,4, Aslihan Dincer (1) ${ }^{3}$, \\ Anastasia P. Grigorenko ${ }^{1,2}$, Gennady Fedonin ${ }^{2}$, Tobias Halene ${ }^{3}$, Maria Aliseychik ${ }^{2,4}$, Elena Filippova', Zhiping Weng (1) ${ }^{5}$, \\ Schahram Akbarian (1), and Evgeny I. Rogaev ${ }^{1,2,4,6}$
}

\begin{abstract}
Both heritability and environment contribute to risk for schizophrenia. However, the molecular mechanisms of interactions between genetic and non-genetic factors remain unclear. Epigenetic regulation of neuronal genome may be a presumable mechanism in pathogenesis of schizophrenia. Here, we performed analysis of open chromatin landscape of gene promoters in prefrontal cortical (PFC) neurons from schizophrenic patients. We cataloged cell-typebased epigenetic signals of transcriptional start sites (TSS) marked by histone H3-K4 trimethylation (H3K4me3) across the genome in PFC from multiple schizophrenia subjects and age-matched control individuals. One of the top-ranked chromatin alterations was found in the major histocompatibility (MHC) locus on chromosome 6 highlighting the overlap between genetic and epigenetic risk factors in schizophrenia. The chromosome conformation capture (3C) analysis in human brain cells revealed the architecture of multipoint chromatin interactions between the schizophrenia-associated genetic and epigenetic polymorphic sites and distantly located HLA-DRB5 and BTNL2 genes. In addition, schizophrenia-specific chromatin modifications in neurons were particularly prominent for non-coding RNA genes, including an uncharacterized LINC01115 gene and recently identified BNRNA_052780. Notably, proteincoding genes with altered epigenetic state in schizophrenia are enriched for oxidative stress and cell motility pathways. Our results imply the rare individual epigenetic alterations in brain neurons are involved in the pathogenesis of schizophrenia.
\end{abstract}

\section{Introduction}

Schizophrenia (SZ) is a complex disorder with highly variable clinical manifestations of psychotic symptoms, such as auditory hallucinations, paranoid or bizarre delusions, disorganized thinking, and significant social dysfunction. Considerably higher concordance in monozygotic twins than in dizygotic twins or siblings indicates that there is a genetic component to this disease. The genetic methods, such as linkage in pedigrees and large scale genome-wide association studies (GWASs) have

\footnotetext{
Correspondence: Evgeny I. Rogaev (evgeny.rogaev@umassmed.edu) ${ }^{1}$ Department of Psychiatry, University of Massachusetts Medical School, Worcester, MA, USA

${ }^{2}$ Department of Human Genetics and Genomics, Laboratory of Evolutionary Genomics, Vavilov Institute of General Genetics of Russian Academy of Science, Moscow, Russia
}

Full list of author information is available at the end of the article been employed to identify the genomic loci contributing to schizophrenia ${ }^{1}$. No robust evidence has been obtained showing the contribution of common genetic variations in the majority cases of SZ. Many studies have identified genomic loci putatively associated with SZ, among which the major histocompatibility complex (MHC) locus, which includes the cluster of human leukocyte antigen (HLA) genes, on chromosome 6, has emerged as the most robust signal in many GWAS studies, along with several other weaker signals on various chromosomes ${ }^{2}$. A recent direct search of rare variations and de novo mutations in protein-encoding gene regions in individuals with SZ revealed no significant overlap for the identified mutant genes in independent studies ${ }^{3-7}$. Collectively, few genetic markers have been discovered, which together explain a small proportion of the estimated genetic component. It is

\section{(c) The Author(s) 2019}

(c) Open Access This article is licensed under a Creative Commons Attribution 4.0 International License, which permits use, sharing, adaptation, distribution and reproduction cc) in any medium or format, as long as you give appropriate credit to the original author(s) and the source, provide a link to the Creative Commons license, and indicate if changes were made. The images or other third party material in this article are included in the article's Creative Commons license, unless indicated otherwise in a credit line to the material. If material is not included in the article's Creative Commons license and your intended use is not permitted by statutory regulation or exceeds the permitted use, you will need to obtain permission directly from the copyright holder. To view a copy of this license, visit http://creativecommons.org/licenses/by/4.0/. 
conceivable that many rare genetic variants with relatively modest or week effects may contribute to schizophrenia phenotype.

The human genome is decorated by numerous chemical modifications, jointly referred as "epigenetics". They include direct modifications of DNA bases (for example, methylation of cytosines) and also changes of histones, which form a nucleosome-a protein complex DNA wraps around. Both of these epigenetic modifications are connected to gene expression ${ }^{8,9}$. For example, H3K4me3 marks promoters of active genes ${ }^{10,11}$. Interestingly, unlike genetic variants, epigenetic modifications are subject to environmental factors and change during lifetime. Therefore, epigenetics may at least partly explain the unexplained proportion of SZ genetic component.

Several studies have explored the epigenetic changes in SZ comparing to controls (CTRL), but their significance in schizophrenia neuropathology is still unclear ${ }^{12-15}$. Some studies investigated the epigenetics of the peripheral blood cells, but the brain epigenomic profiling is more relevant in the context of SZ. In particular, the prefrontal cortex (PFC) plays a major role in cognition and psychiatric diseases. However, brain epigenetic studies are complicated due to the heterogeneity of brain tissues, which contain many different cell types. While recently emerged single-cell epigenomic profiling is a very promising approach ${ }^{16}$, sorting and analysis of whole specific cell population allow us to investigate larger casecontrol cohorts. For, example RNA binding protein fox-1 homolog $3(\mathrm{NeuN})$ can be used to select neuronal nuclei specifically and exclude non-neuronal brain cells from analysis $^{17-20}$.

Using epigenomic profiling of $\mathrm{H} 3 \mathrm{~K} 4 \mathrm{me} 3$, which is highly regulated in the developing and mature $\mathrm{PFC}^{17}$, we performed outlier analysis and explored epigenetic chromatin variations in cortical neurons specific for patients with SZ. We identified rare specific epigenetic variations in neurons of schizophrenia patients. The enrichment for rare up- or down open chromatin signals in schizophrenia were found for a set of novel non-coding RNA genes and genes essential in certain cellular pathways. We hypothesize that such rare epigenetic variants may affect regulation of gene expression and contribute to pathogenesis of SZ.

\section{Materials and methods H3K4me3 ChIP-seq data}

We analyzed whole-genome ChIP-seq data obtained for chromatin regions marked by H3K4me3-specific antibodies in dorsolateral PFC neurons extracted from postmortem brain samples in a cohort of 16 unrelated patients with SZ and 16 age-matched CTRL individuals. For the accurate comparative analysis the identical experimental and bioinformatics protocols were used to generate ChIPseq data for SZ and CTRL ${ }^{17-20}$ (Supplementary Table 1).

\section{Bioinformatic analysis}

For ChIP-seq data analysis, we used ChIP-seq reads mapped to the human reference genome GRCh37 with Bowtie $2^{21}$ using default parameters. We applied MACS $1.4 .3^{22}$ to identify 29,547 loci of H3K4me3 signal enrichment in human prefrontal neuronal samples from a cohort that includes a subset of SZ individuals ${ }^{23}$ (with $P$-values of less than $1 \times 10^{-10}$ against an input sample) and simultaneously generate coverage tracks for each sample. To account for unequal sequencing depth, we first generated a list of promoter regions for known genes from Ensembl v87 annotation (we collected transcriptional start sites (TSSs) for all transcripts, generated $4 \mathrm{kbp}$ regions centered in TSSs and merged overlapping regions), for each sample tallied the total number of reads mapped to these regions, divided this tally by $10^{7}$, and then normalized the coverage at each base pair by the resulting number. Finally, for each sample we quantified the coverage in each of 29,547 regions as the sum of normalized coverages at each base pair of a particular region.

In order to detect regions enriched in H3K4me3 in samples from patients with SZ against all CTRLs, we selected upregulated peaks for which at least one individual with SZ had at least a 1.5-fold change compared to every of 16 CTRLs and with normalized coverage of at least 5. For downregulated peaks, we selected peaks for which at least one patient with SZ had at most a 0.5 -fold change compared to every of 16 CTRLs and every CTRL have a normalized coverage of at least 5 . In addition, we computed the nominal $P$-value for each peak individually as the probability of drawing the SZ coverage value for normal distributions with parameters estimated from all CTRL coverage values and required it to be less than 0.01 . In both analyses, we also excluded peaks with low mapping quality reads (if $75 \%$ of reads in the peak had MAPQ $<10)$ and loci with low normalized coverage $(<2.5)$. The stringent threshold of fold change compared to all CTRL individuals provides diminished positive rate while missing signals are expected (false negatives).

For both DAVID ${ }^{24}$ and ConsensusPathDB ${ }^{25}$ analyses, we selected genes with TSSs of protein-coding transcripts in close proximity $(<2 \mathrm{~kb})$ to one of the altered peaks. We set false discovery rate threshold at 0.05 for this analysis.

Intersections of genetic datasets for GWASs, exome data, and de novo mutations with epigenetic datasets for H3K4me3 variations in individuals with SZ were analyzed. For GWASs, we obtained raw $P$-values for all evaluated variants and generated genomic sets by selecting all variants with $P$-value $<5 \times 10^{-8}$ and including 100 -kb flanking regions. To evaluate significance of overlap, we used 
H3K4me3 peaks that overlapped with these genomic sets and the whole genetic variant set evaluated in the GWAS as a background. For exome (both case-control and de novo mutations) studies, we selected all SZ-altered peaks close $(2 \mathrm{~kb})$ to the TSSs of protein-coding genes reported in corresponding studies. We used all protein-coding genes as a background in this analysis. For CNVs, we used the whole genome as a background. For SZDB analysis, we also used all protein-coding genes as a background. We applied one-tailed Fisher's exact test to evaluate statistical significance for overlap of up-, down- and all peaks for both SZ14 and SZ2 groups separately (six tests per each dataset) with false discovery rate $<0.05$.

\section{Chromosomal conformation capture at the HLA-DRB9 locus}

Postmortem PFC brain tissues for three SZ cases and three CTRLs were obtained from the University of Maryland and pair-matched for age, sex, postmortem interval, and $\mathrm{pH}$ (Supplementary Table 2). In order to detect DNA looping interactions 3C libraries were prepared as previously described ${ }^{26}$. $3 \mathrm{C}$ primers (Supplementary Table 3) were designed based on the following criteria: (i) distance less than $200 \mathrm{bp}$ from the HindIII restriction site; (ii) $30 \mathrm{bp}$ in length; (iii) $\mathrm{GC} \%$ of $40-45 \%$; and (iv) melting temperature between 59 and $62^{\circ} \mathrm{C}$.

\section{Results}

\section{Genomic regions showing outlier chromatin changes marked by $\mathrm{H} 3 \mathrm{~K} 4 \mathrm{me} 3$ in SZ}

We performed comparative analysis of datasets of H3K4me3 profiling in PFC neurons from patients with SZ $(N=16)$ and CTRL individuals $(N=16$; Supplementary Table 1). Genome-wide correlation analysis demonstrated that H3K4me3 profiles of two unrelated patients with SZ (S10 and S11) were different (mean Pearson correlation coefficient, $\mathrm{PCC}=0.89$ ) from all other individuals but showed much higher similarity to each other $(\mathrm{PCC}=0.97$; Supplementary Figure 1). No particular clinical phenotypes or treatment specifically shared by these two subjects have been detected in available medical records. To exclude potential unknown confounding factors, we separately analyzed these two samples (SZ2 group) and another 14 individuals (SZ14 group).

To evaluate the roles of rare H3K4me3 variations, we searched for loci with significant individual alterations in patients with SZ with at least 1.5-fold changes in particular SZ individual compared to all control individuals (Fig. 1) to focus on the most robust individual epigenetic variants. For the SZ14 group we found 561 different genomic loci, with 227 upregulated loci and 335 downregulated loci in SZ (one locus was simultaneously upregulated in patient S8 and downregulated in patient S6; Supplementary Table 4, Supplementary Figure 2). In the SZ14 group, most of the upregulated or downregulated loci $(89 \%)$ were observed in a single patient only, thereby representing individual-specific alterations. However, there were 56 loci (22 upregulated loci and 34 downregulated loci) with simultaneous alterations in at least two samples. For the SZ2 group, we found 1,265 loci (1,003 upregulated loci and 262 downregulated loci; Supplementary Table 5, Supplementary Figure 3) with 196 (15\%) regions simultaneously altered in both samples.

A notable example in the SZ14 group was upregulation of the H3K4me3 peak (Fig. 1a, j) for individuals with SZ on chromosome 2 harboring TSS for two ncRNA genes, i.e., LINCO1115 and the novel unannotated previously gene BNRNA_052780, which is in a group of novel ncRNA genes that we have recently discovered ${ }^{23}$. Thus, this analysis revealed a single common neuronal marker shared by up to $50 \%$ of individuals with SZ, which formed a homogenous group in genome-wide correlation analysis of H3K4me3 profiles. In addition to the LINCO1115/ BNRNA_052780 locus, the top upregulated locus was identified in the $3^{\prime}$ end of $H L A-D R B 9$ (Fig. 2a) altered in three patients with SZ. The most robust downregulated loci included a locus on chromosome 19 in close proximity to the ncRNA RP11-678G14.3 (Fig. 1b) and on chromosome 22, with the predicted novel ncRNA gene, BNRNA_062360 (Fig. 1c). Each of these two downregulated loci was altered in four individuals with SZ.

\section{The epigenetic roles of the HLA-locus and immunogenes in neurons}

One of the two top H3K4me3 upregulated loci in SZ (the SZ peak) was identified in the $3^{\prime}$ end of the $H L A$ $D R B 9$ pseudogene located between the HLA-DRA and $H L A-D R B 5$ genes (Fig. 2a). This upregulation was found in at least three men with SZ (patients S8, S9, and S14), ages 50,45 , and 22 years old, respectively, and tended to be male-specific (Fisher's exact test nominal $P$-value $=$ 0.02). Moreover, this locus was strongly marked by H3K4me3 in the testis (Fig. 2b).

Interestingly, this peak was close to a cluster of single nucleotide polymorphisms (SNPs), which are strongly associated with $\mathrm{SZ}^{27}$ (Fig. 2c). Among these SNPs, rs9268895 has been previously reported to have the strongest association with SZ across the whole genome (under identifier rs114002140) with a nominal discovery $P$-value $8.28 \times 10^{-1528}$. We found that another SNP (rs9268830) is located within the H3K4me3 SZ-peak, and its $G$ allele showed strong linkage to the A allele of rs9268895 $\left(D^{\prime}=0.964\right.$, Supplementary Figure $\left.4 \mathrm{~b}\right)$. This H3K4me3 peak, located in the $3^{\prime}$ region of the HLA-DRB9 gene, is far from any TSS for any known gene in this region $(>14 \mathrm{~kb})$. To elucidate the presumable chromatin interactions in this locus, we performed $3 \mathrm{C}$-analysis in human brain samples. We anchored primers at the 


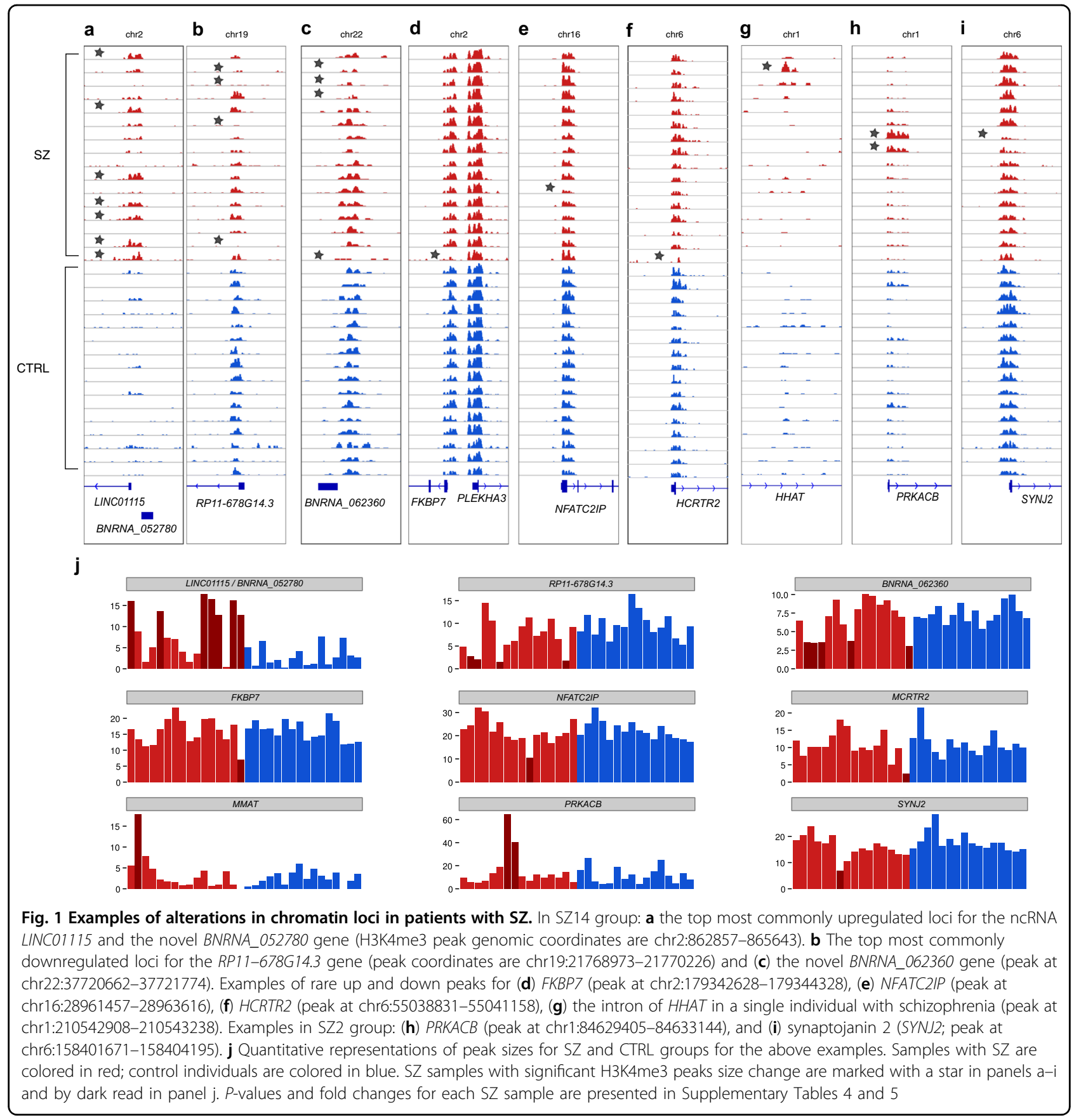

H3K4me3 peak, the GWAS significant SNP rs9268895, promoter of the distantly located BTNL2 gene, and the TSS of the pseudogene HLA-DRB9 to test for physical looping interactions with genes in the region in pairmatched postmortem PFC samples from individuals diagnosed with SZ and CTRLs (Fig. 3a, Supplementary Tables 2, 3). We detected four sequence-verified interactions: (1) between the SZ-peak and BTNL2; (2) between the SZ-peak and rs9268895; (3) between the SZ-peak and the HLA-DRB9 TSS; and (4) between rs9268895 and
HLA-DRB5 (Fig. 3b). Each looping interaction was identified by sequencing in at least one of the tested individual brain specimens. Our data indicated that the H3K4me3marked region in the pseudogene $H L A-D R B 9$ formed a physical loop with nearby genes BTNL2 and HLA-DRB5.

In addition to the HLA locus, immune response gene regions are the most commonly genetic loci associated with SZ and other psychiatric illnesses in GWASs ${ }^{29,30}$. Here, we made interesting observation. We found that at least 651 (51\%) of 1289 known immunogenes 

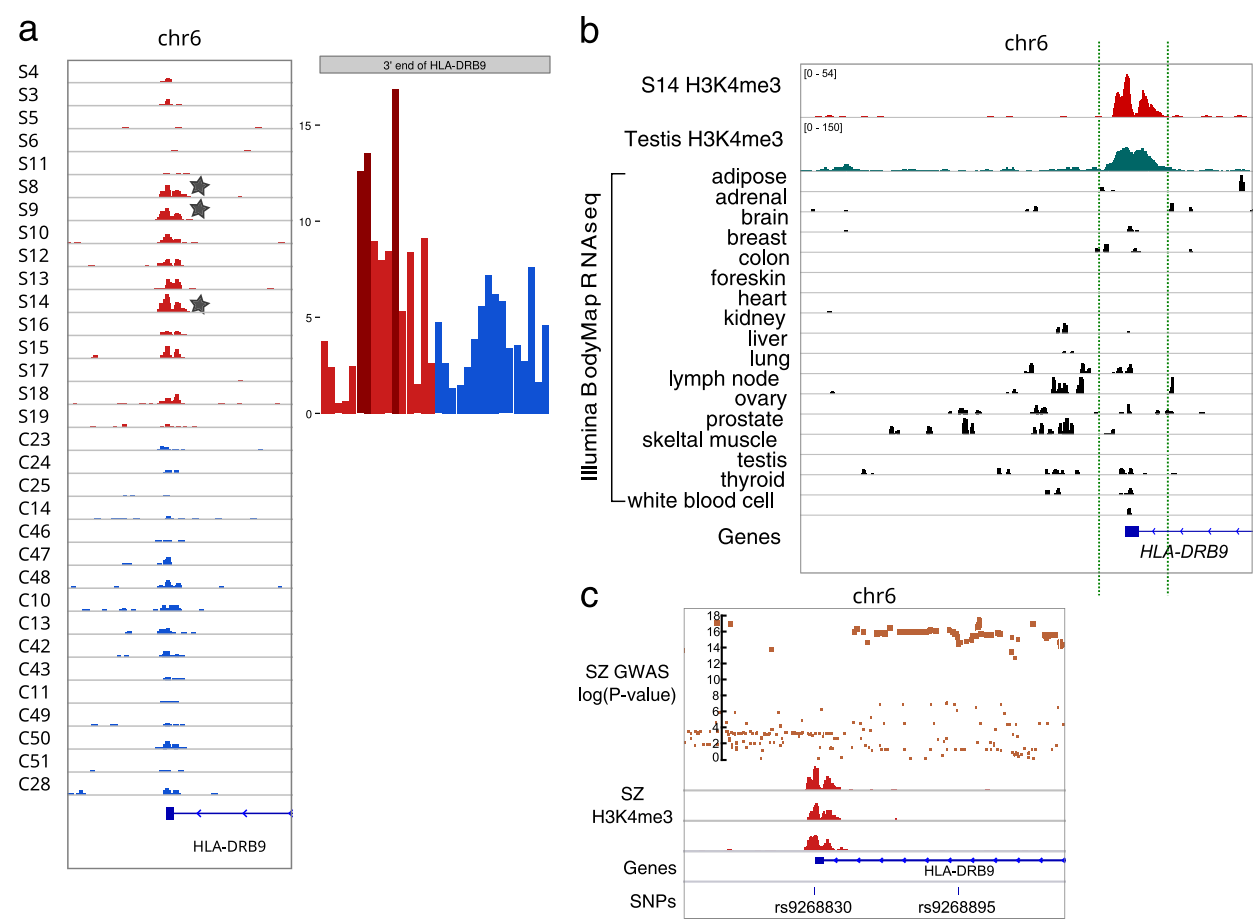

Fig. 2 Analysis of the HLA-DRB9 locus. a The H3K4me3-marked open chromatin peak at the distal 3'-region of HLA-DRB9 was upregulated in neurons from individuals with schizophrenia (indicated by a star); peak genomic coordinates are chr6:32427120-32428371. b The transcription activity(lllumina BodyMap 2.0 data) and chromatin state ${ }^{62}$ suggest common activity of this locus in the testis. $\mathbf{c}$ A cluster of significant variations from GWAS data for schizophrenia next to the peak (18). The log Y-scale represents reported nominal $P$-values for variants (36,989 cases and 113,075 controls). Bottom panel: the SNPs rs9268830 (inside the peak) and rs9268895 (the most significant SNP associated with schizophrenia in GWAS data) (19). SZ samples are marked with S prefix, control individuals are marked with C prefix. Red and blue colors indicate SZ and CTRL, respectively

(Supplementary Note) showed the active H3K4me3marked chromatin state in human prefrontal cortex neurons. Among these genes, $13(2.0 \%)$ and $30(4.6 \%)$ were dysregulated in the SZ14 and SZ2 groups, respectively (Supplementary Table 6).

These data suggested that immunogenes may have a broad and direct role in neuronal function or presumable epigenetic responses of neurons to inflammatory immune factors.

\section{Gene ontology and molecular pathways for epigenetically modified SZ loci}

To further elucidate whether protein-coding genes with promoters marked by up- or downregulated loci in patients with SZ were enriched for any particular biological pathways or gene ontology terms ${ }^{31}$, we used DAVID $^{24}$ and ConsensusPathDB ${ }^{25}$ web services for analysis of SZ-associated H3K4me3 loci.

In the SZ14 group, ConsensusPathDB analysis (Supplementary Table 7a) showed the strongest enrichment for genes involved in response to reactive oxygen species (false discovery rate (FDR)-adjusted $P=1.67 \times 10^{-5}$ ) and regulation of cell motility (FDR-adjusted $P=3.12 \times 10^{-5}$; Fig. 4a). For example, the gene for platelet-derived growth factor receptor, beta polypeptide (PDGFRB), which modulates the production of reactive oxygen species via NADPH oxidases ${ }^{32}$, is down-regulated in subject S13. The gene for one of multimerin 2 (MMRN2) isoforms, which regulates cell motility, is up-regulated in subject $\mathrm{S} 12$. This gene is located within a genomic region harboring 10q22-23 deletions, which are associated with behavioral abnormalities ${ }^{33,34}$. The pathways for response to reactive oxygen species and regulation of cell motility have been associated with schizophrenia: there is evidence of oxidative damage in $\mathrm{SZ}^{35-38}$ and cell motility is driven by actin filament dynamics ${ }^{39}$, which have been linked to de novo mutations in a recent study of patients with $\mathrm{SZ}^{40}$. DAVID analysis revealed no statistically significant enrichment. However, the top term was the phospholipase C (PLC) pathway, particularly hypocretin (orexin, $H C R T)$ and hypocretin receptor 2 (HCRTR2), in a few individuals with SZ (Supplementary Figure 2, Supplementary Table 8; see Discussion).

In the SZ2 group, enrichment analysis demonstrated remarkable enrichment for the terms synapse (FDRadjusted $P=2.1 \times 10^{-7}$ ) and cell junction (FDR-adjusted $P=4 \times 10^{-7}$ ) using DAVID (Supplementary Table 9); with ConsensusPathDB (Fig. 4b, Supplementary Figure 1l, 

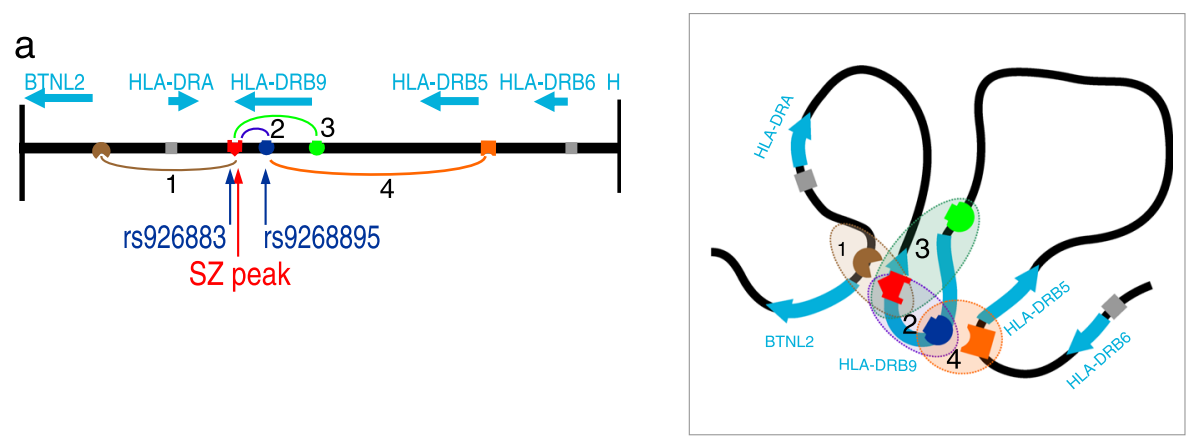

b
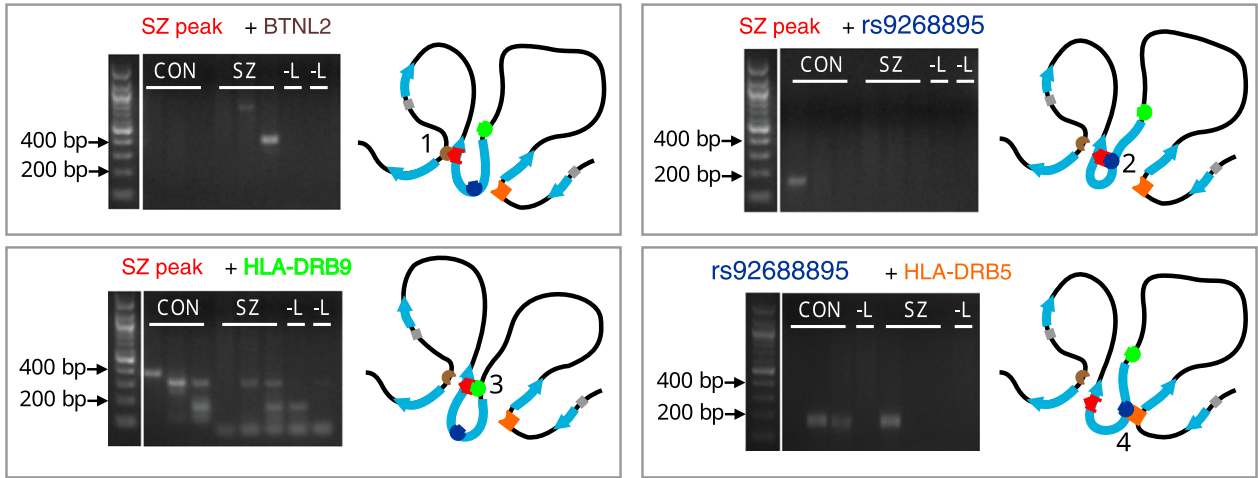

Fig. 3 Putative looping interactions in the HLA-DRB9 locus. a Schematic representation of genes near the peak and putative looping interactions between SZ peak, SNP rs9268895, and nearby genes BTNL2, HLA-DRB9, and HLA-DRB5. b 3C experimental analysis of cells from postmortem cortical tissues revealed at least four looping interactions. SZ schizophrenia; CON control; - $L$ no ligase

Supplementary Table $7 \mathrm{~b})$, the top terms were nervous system development (FDR-adjusted $P=2.36 \times 10^{-6}$ ) and cell differentiation (FDR-adjusted $P=9.38 \times 10^{-5}$ ). Overall, these terms involved cadherins and their interacting partners, and some were markedly dysregulated in patients S10 and S11 (Supplementary Figure 3a-c). The difference in enrichment of gene pathways and ontology categories between SZ2 and SZ14 groups supports the global epigenetic reprogramming of genes for certain pathways in the prefrontal neurons in the two SZ2 samples. Although unknown confounding effects cannot yet be completely ruled out, these epigenetic differences may present a phenomenon of biological heterogeneity of the psychiatric disorder defined clinically under common SZ patterns.

Notably, we linked only $48 \%$ and $60 \%$ of SZ-altered loci to known protein-coding genes in the SZ14 and SZ2 groups, respectively, indicating that, correspondingly, 52\% and $40 \%$ of regions were not included in the pathway analysis. Consistent with this, altered peaks were enriched for ncRNA genes in the SZ14 group (Bonferroni adjusted $P$-value $=0.007$, Table 1 ). Thus, ncRNA genes may have a broader role than protein-coding genes in epigenetic alterations in SZ pathogenesis. Overall, we identified novel loci showing alterations in active chromatin for
ncRNA genes, which may be involved in SZ. However, the functions of these alterations have not been evaluated yet.

\section{Integration of ChIP-seq data with high-throughput genetic studies}

Next, we sought to test whether the H3K4me3 loci altered in neurons of individuals with SZ were enriched in the dataset of disease loci found in high-throughput genetic studies. The same genetic risk factors can contribute to different psychiatric diseases, particularly in SZ and affective disorders (e.g., bipolar disorder [BD] and major depressive disorder $[\mathrm{MDD}])^{41}$. Therefore, we downloaded the GWAS dataset reported for SZ (Schizophrenia Working Group, SWG set: 36,989 individuals with SZ and 113,075 CTRLs) ${ }^{27}$ and the GWAS data for five major psychiatric disorders (Cross-Disorder Group, GDG set: 33,332 cases and 27,888 controls) ${ }^{41}$. We investigated the overlap between H3K4me3 loci altered in SZ and the lists of SNPs (and the flanking 200-kb region) associated with psychiatric diseases. The SNP-associated loci were selected as sites with significant (nominal $P<$ $5 \times 10^{-8}$ ) and suggestive (nominal $P<1 \times 10^{-6}$ ) thresholds (Supplementary Tables 10, 11). Comparatively, recent brain DNA methylation studies of SZ have shown weak ${ }^{13}$ or no ${ }^{42}$ direct overlap with the same SWG data. We also 


$\begin{array}{ll}\text { Gene Ontology category } & -\log _{10} \text { (FDR-adjusted P-value) } \\ \text { response to reactive oxygen species } & 2.20 \\ \text { regulation of cell motility } & 2.20 \\ \text { regulation of cellular component movement } & 1.91 \\ \text { positive regulation of locomotion } & 1.91 \\ \text { cellular response to organic substance } & 1.89 \\ \text { regulation of epithelial cell migration } & 1.87 \\ \text { response to steroid hormone } & 1.87 \\ \text { positive regulation of cellular process } & 1.79 \\ \text { positive regulation of homeostatic process } & 1.48 \\ \text { response to lipid } & 1.48\end{array}$

Fig. 4 Top 10 enriched gene ontology terms analyzed with ConsensusPathDB for the SZ14 group. Terms with FDR-adjusted $P$-values of less than 0.01 are highlighted in green

found a relatively modest or no sign of overlap for the ChIP-seq and GWAS signals for psychiatric illnesses (Supplementary Tables 10 and 11). We found nominally significant overlap of epigenetic up- or downregulated H3K4me3 loci and loci harboring SNPs with suggestive associations (GWAS $P<1 \times 10^{-6}$ ) with major depression disorder (MDD) in the GDG set for the SZ14 $(P=0.04)$ groups (Supplementary Table 11). In total, no significant enrichment was found for a set of epigenetic SZ loci and a set of multiple genetic loci putatively associated in GWASs with schizophrenia, autism spectrum disorder, attention deficit-hyperactivity disorders, or diabetes used as a control dataset (Supplementary Table 11) for the SZ14 group. We also found that, on a global level, the list of SZ-associated H3K4me3 loci was not significantly enriched by copy number variation $(\mathrm{CNV})$ regions or by genes bearing rare de novo or inherited mutations found in $\mathrm{SZ}^{3,5,6,40,43}$.

However, we found some specific genes showing overlap between our set of genes with epigenetic changes and a set genes for genetic risk for SZ and other psychiatric disorders (Supplementary Table 10). For example, the genes encoding L-type voltage-gated calcium channel subunits and dipeptidase 2 (DPEP2) were identified in many GWASs or in both GWASs and exome sequencing studies of SZ data (Supplementary Figure 2c). Interestingly, among the top seven up- and down-regulated H3K4me3 loci listed in the SZ14 group, at least two loci (HLA and TRAF3IP2) were strongly associated with SZ in SWG data, and a third locus harboring the ncRNA genes was assigned to 22q13.1, the chromosomal locus showing genetic association
Table 1 Enrichment of loci characterized by up and downregulated $\mathrm{H} 3 \mathrm{~K} 4 \mathrm{me} 3$ peaks in schizophrenia in genomic regions harboring non-coding RNA genes or regions with no Ensembl annotated genes

\begin{tabular}{|c|c|c|c|}
\hline \multirow[t]{2}{*}{ H3K4me3 peak category } & \multirow[t]{2}{*}{$\begin{array}{l}\text { Total } \\
\text { peak count }\end{array}$} & \multicolumn{2}{|c|}{$\begin{array}{l}\text { SZ14 up- and down- } \\
\text { peaks (561) }\end{array}$} \\
\hline & & Count & $\begin{array}{l}\text { Bonferroni } \\
\text { adjusted P- } \\
\text { value }\end{array}$ \\
\hline Ensembl annotated genes & 20546 & 269 & 1.0 \\
\hline Protein-coding genes & 16689 & 157 & 1.0 \\
\hline Non-coding genes & 3857 & 112 & 0.00006 \\
\hline $\begin{array}{l}\text { No Ensembl annotated } \\
\text { genes (includes novel } \\
\text { neuronal ncRNA genes }{ }^{23} \text { ) }\end{array}$ & 9000 & 192 & $1 \times 10^{-25}$ \\
\hline
\end{tabular}

with SZ and abnormal behavior-associated 22q13 deletion syndrome ${ }^{44}$.

While globally the overlap with genetic data was not significant, when we used 2,706 genes from the SZDB database $^{45}$, which includes not only GWAS data but also genetic linkage, gene expression, protein interaction, and convergent function genomics data, we found a nominally significant overlap with up-regulated peaks in the SZ14 group (nominal $P=0.007$ ) (Supplementary Table 12). The overlap between differentially expressed genes from SZDB and epigenetically modified genes in SZ from the SZ14 group included the LINC01115 ncRNA gene. The observed overlap supported the role of the identified loci and genes in SZ etiology.

\section{Discussion}

In this study, we performed an outlier analysis of highresolution mapping of the open chromatin mark, H3K4me3, which is primarily associated with active gene promoters, in PFC neurons of patients with SZ and CTRLs.

We identified multiple loci with up- and downregulated open chromatin in PFC neurons of patients with SZ versus CTRL individuals. For example, a locus in the promoter of the ncRNA LINCO1115 showed elevated H3K4me3 in half of our small set of schizophrenic patients. According to SZDB ${ }^{45}$, LINC01115 RNA transcript was also reported to be upregulated in the PFC of patients with SZ in one study ${ }^{46}$. The function of LINC01115 is poorly characterized. This gene is located $\sim 100 \mathrm{~kb}$ upstream of TMEM18, which is well-known to associate with obesity ${ }^{47}$. A SNP in LINC01115 has been reported to epistatically interact with $T M E M 18^{48}$. A methylation state of the CpG region located in 
LINC01115 is linked to fat mass and fat free mass ${ }^{49}$. These reports suggest that the LINC01115 gene/locus may regulate TMEM18 expression. Body composition per se is altered in $\mathrm{SZ}^{50}$. Most interestingly, TMEM18 modulates the migration of neuronal precursor cells and neural stem cells $^{51}$. Notably, abnormal migration of neuronal progenitor cells has was also been reported for schizophrenia subjects $^{52}$. Therefore, our analysis revealed a single putative neuronal marker for the gene, which may be involved in neural cell migration, shared by up to $50 \%$ of schizophrenic individuals with SZ in our sample set. Additional studies are needed to confirm these findings in a larger cohort.

Using gene ontology analysis, we found that the groups of genes with up- and downregulation of H3K4me3 loci were statistically significantly enriched with genes for oxidative response and regulation of cell motility in the SZ14 group. In addition, using DAVID, we observed the enrichment for the plasma membrane genes and cell motility in the top categories for the SZ14 group; however, these results were not statistically significant. Remarkably, regulation of phospholipase activity was one of the top pathways enriched in the downregulated loci for SZ. Phosphoinositide-phospholipase C- $\beta 1$ (PLC- $\beta 1$ ) is abnormally expressed in $\mathrm{SZ}^{53,54}$ and knockout of PLC- $\beta 1$ in mice results in SZ-like phenotypes ${ }^{55}$. Within this pathway, receptor 1 of sphingosine 1-phosphate (S1PR1) is involved in activation of the PLC pathway ${ }^{56}$, and activation of HCRT and HCRTR2 increases PLC activity ${ }^{57}$. We found these genes downregulated in some patients with SZ.

Two patients with SZ in our dataset showed global chromatin alterations, particularly for synaptic genes, that were distant from those in other individuals with SZ. These data were consistent with recent independent observations of differential DNA methylation for some patients with SZ, which was very distant from others in SZ

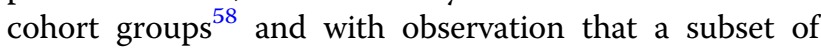
schizophrenia subjects form a separate group based on global alteration of transcriptome ${ }^{59}$. The segregation of SZ2 subjects from SZ14 group may be explained by distant etiology or symptomatology of SZ subtypes. Alternatively, it might reflect the drug effect that robustly changed the epigenomic state in SZ2 subjects ${ }^{60}$. To address this we showed no mix-up for the SZ2 samples that belong two independent subjects and that $\mathrm{H} 3$ K4me3 signal alterations overlap with genes showing change in expression under pharmacological induction and treatment of schizophrenia-like symptoms in primate-model (Supplementary Note, Supplementary Table 13) ${ }^{61}$.

Our data reveal no overlap between epigenetically modified genes and genes bearing reported de novo mutations associated with schizophrenia ${ }^{38}$. Additional studies involving large cohorts of individuals with SZ need to be performed in order to estimate the total contributions of de novo mutations in H3K4me3-marked regions at risk for SZ. In total, our data demonstrated no global overlap between the loci showing H3K4me3 up- and down-regulation in schizophrenic subjects and schizophrenia-associated genetic loci implied in GWAS studies.

However, our results revealed a set of the genes showing both neuronal chromatin alterations and genetic associations with SZ. Notably, the region within the HLA gene cluster in the MHC locus on chromosome 6, which is the strongest and most common genetic risk factor for $\mathrm{SZ}^{27}$, also appeared to be a top epigenetically modified locus in $\mathrm{SZ}$ in this study. Furthermore, we demonstrated a potential physical looping interaction between identified region and nearby genes (HLA-DRB5 and BTNL2), which suggests a role of novel promoter located in $H L A-D R B 9$ pseudogene or for non-promoter effect of this H3K4me3 signal in cortical neurons. However, if and how these DNA interactions are associated with SZ have yet to be studied.

Overall, in this study we demonstrated, that most of the epigenetic variations found specifically in individuals with SZ were rare and enriched at loci harboring non-coding genes. Interestingly, that the top H3K4me3 marked loci are also reported in top of genetic loci associated with schizophrenia (HLA locus on chromosome 6 and TRAF3IP2 locus on chromosome 22) suggesting a potential causative role for such epigenetic changes. Given that many epigenetic changes are rare or private in the schizophrenia subjects they may represent random epigenetic variations that can be termed epi-mutations. We can hypothesize that such rare epi-mutations occurring in neurons could contribute to risk for schizophrenia. On the other hand, given the partial overlap for the global epigenetic alterations shared by some subjects, the subset of epigenetic signals can be a result of certain conditions associated with schizophrenia pathogenesis or environment, e.g. specific changes induced by drug therapy affecting neurotransmitter signaling. Finally, the study presents the list of novel ncRNA genes with epigenetic changes in schizophrenia, providing the new insight for further study of schizophrenia mechanisms.

\footnotetext{
Acknowledgements

This work was supported, in part, by NIH (grant no. AG054712) to E.I.R., the Brain \& Behavior Research Foundation, Autism Speaks, and NIMH (grant no. MH106056) to S.A., A.M., and A.D. Russian Science Foundation (RSF; grant no. 14-44-00077) supported, in part, F.E.G., T.V.A., M.A., bioinformatic analysis of ChIP-seq data; and grant no. 19-75-30039 supported, in part, F.E.G, T.V.A, M.A., gene ontology analysis. Russian Foundation for Basic Research (RFBR, grant no. 18-29-13051) supported, in part, F.E.G, T.V.A., analysis of large scale genetic datasets.
} 


\section{Author details}

'Department of Psychiatry, University of Massachusetts Medical School, Worcester, MA, USA. ²Department of Human Genetics and Genomics, Laboratory of Evolutionary Genomics, Vavilov Institute of General Genetics of Russian Academy of Science, Moscow, Russia. ${ }^{3}$ Department of Psychiatry and Department of Neuroscience, Friedman Brain Institute, Icahn School of Medicine at Mount Sinai, New York, NY, USA. ${ }^{4}$ Faculty of Biology, Center for Genetics and Genetic Technologies, Faculty of Bioengineering and Bioinformatics, Lomonosov Moscow State University, Moscow, Russia. ${ }^{5}$ Program in Bioinformatics and Integrative Biology, University of Massachusetts Medical School, Worcester, MA, USA. 'Sirius University of Science and Technology, 1 Olympic Ave, 354340 Sochi, Russia

\section{Conflict of interest}

The authors declare that they have no conflict of interest.

\section{Publisher's note}

Springer Nature remains neutral with regard to jurisdictional claims in published maps and institutional affiliations.

Supplementary Information accompanies this paper at (https://doi.org/ 10.1038/s41398-019-0596-1).

Received: 15 June 2019 Revised: 9 September 2019 Accepted: 24 September 2019

Published online: 17 October 2019

\section{References}

1. Insel, T. R. Rethinking schizophrenia. Nature 468, 187-193 (2010).

2. De Jong, S. et al. Expression QTL analysis of top loci from GWAS meta-analysis highlights additional schizophrenia candidate genes. Eur. J. Hum. Genet. 20, 1004-1008 (2012)

3. Kirov, G. et al. De novo CNV analysis implicates specific abnormalities of postsynaptic signalling complexes in the pathogenesis of schizophrenia. Mol. Psychiatry 17, 142-153 (2012).

4. Guha, S. et al. Implication of a rare deletion at distal 16p11.2 in schizophrenia. JAMA Psychiatry 70, 253-260 (2013).

5. Purcell, S. M. et al. A polygenic burden of rare disruptive mutations in schizophrenia. Nature 506, 185-190 (2014).

6. Szatkiewicz, J. P. et al. Copy number variation in schizophrenia in Sweden. Mol. Psychiatry 19, 762-773 (2014).

7. Loohuis, L. M. et al. Genome-wide burden of deleterious coding variants increased in schizophrenia. Nat. Commun. 6, 7501 (2015).

8. Kass, S. U., Landsberger, N. \& Wolffe, A. P. DNA methylation directs a timedependent repression of transcription initiation. Curr. Biol. 7, 157-165 (1997).

9. Tropberger, P. \& Schneider, R. Scratching the (lateral) surface of chromatin regulation by histone modifications. Nat. Struct. Mol. Biol. 20, 657-661 (2013)

10. Pradeepa, M. M. et al. Histone H3 globular domain acetylation identifies a new class of enhancers. Nat. Genet. 48, 681-686 (2016).

11. Kouzarides, T. Chromatin modifications and their function. Cell 128, 693-705 (2007).

12. Bryois, J. et al. Evaluation of chromatin accessibility in prefrontal cortex of individuals with schizophrenia. Nat. Commun. 9, 3121 (2018).

13. Jaffe, A. E. et al. Mapping DNA methylation across development, genotype and schizophrenia in the human frontal cortex. Nat. Neurosci. 19, 40-47 (2015).

14. Aberg, K. A. et al. Methylome-wide association study of schizophrenia: identifying blood biomarker signatures of environmental insults. JAMA Psychiatry 71, 255-264 (2014)

15. Alelú-Paz, R. et al. Epigenetics in schizophrenia: a pilot study of global dna methylation in different brain regions associated with higher cognitive functions. Front. Psychol. 7, 1496 (2016).

16. Clark, S. J. et al. Single-cell epigenomics: powerful new methods for understanding gene regulation and cell identity. Genome Biol. 17, 72 (2016).

17. Shulha, H. P. et al. Human-specific histone methylation signatures at transcription start sites in prefrontal neurons. PLoS Biol. 10, e1001427 (2012).

18. Shulha, H. P. et al. Epigenetic signatures of autism: trimethylated H3K4 landscapes in prefrontal neurons. Arch. Gen. Psychiatry 69, 314-324 (2012).
19. Shulha, H. P. et al. Coordinated cell type-specific epigenetic remodeling in prefrontal cortex begins before birth and continues into early adulthood. PLOS Genet. 9, e1003433 (2013).

20. Mitchell, A. C. et al. MEF2C transcription factor is associated with the genetic and epigenetic risk architecture of schizophrenia and improves cognition in mice. Mol. Psychiatry 23, 123-132 (2018).

21. Langmead, B. \& Salzberg, S. L. Fast gapped-read alignment with Bowtie 2. Nat. Methods 9, 357-359 (2012).

22. Zhang, Y. et al. Model-based analysis of ChIP-Seq (MACS). Genome Biol. 9, R137 (2008).

23. Gusev, F. E. et al. Epigenetic-genetic chromatin footprinting identifies nove and subject-specific genes active in prefrontal cortex neurons. Faseb. J. 33, 8161-8173 (2019)

24. Huang, D. W., Sherman, B. T. \& Lempicki, R. A. Bioinformatics enrichment tools: paths toward the comprehensive functional analysis of large gene lists. Nucleic Acids Res. 37, 1-13 (2009).

25. Kamburov, A., Stelzl, U., Lehrach, H. \& Herwig, R. The ConsensusPathDB interaction database: 2013 Update. Nucleic Acids Res. 41, D793-D800 (2013).

26. Mitchell, A. C. et al. The genome in three dimensions: a new frontier in human brain research. Biol. Psychiatry 75, 961-969 (2014).

27. Ripke, S. et al. Biological insights from 108 schizophrenia-associated genetic loci. Nature 511, 421-427 (2014).

28. Ripke, S. et al. Genome-wide association study identifies five new schizophrenia loci. Nat. Genet. 43, 969-978 (2011).

29. Jia, P., Wang, L., Meltzer, H. Y. \& Zhao, Z. Common variants conferring risk of schizophrenia: a pathway analysis of GWAS data. Schizophr. Res. 122, 38-42 (2010).

30. O'dushlaine, C. et al. Psychiatric genome-wide association study analyses implicate neuronal, immune and histone pathways. Nat. Neurosci. 18, 199-209 (2015).

31. Blake, J. A. et al. Gene ontology consortium: going forward. Nucleic Acids Res. 43, D1049-D1056 (2015).

32. Kreuzer, J. et al. Platelet-derived growth factor activates production of reactive oxygen species by $\mathrm{NAD}(\mathrm{P}) \mathrm{H}$ oxidase in smooth muscle cells through $\mathrm{Gi1}, 2$. FASEB J.: Off. Publ. Federation Am. Societies Exp. Biol. 17, 38-40 (2003).

33. Balciuniene, J. et al. Recurrent 10q22-q23 deletions: a genomic disorder on $10 \mathrm{q}$ associated with cognitive and behavioral abnormalities. Am. J. Hum. Genet. 80, 938-947 (2007).

34. Lorenzon, E. et al. MULTIMERIN2 impairs tumor angiogenesis and growth by interfering with VEGF-ANEGFR2 pathway. Oncogene 31, 3136-3147 (2012).

35. Flatow, J., Buckley, P. \& Miller, B. J. Meta-analysis of oxidative stress in schizophrenia. Biol. Psychiatry 74, 400-409 (2013).

36. Jiang, Z., Cowell, R. M. \& Nakazawa, K. Convergence of genetic and environmental factors on parvalbumin-positive interneurons in schizophrenia. Front. Behav. Neurosci. 7, 116 (2013).

37. Koga, M., Serritella, A. V., Sawa, A. \& Sedlak, T. W. Implications for reactive oxygen species in schizophrenia pathogenesis. Schizophr. Res. 176, 52-71 (2016).

38. Hardingham, G. E. \& Do, K. Q. Linking early-life NMDAR hypofunction and oxidative stress in schizophrenia pathogenesis. Nat. Rev. Neurosci. 17, 125-134 (2016).

39. Pollard, T. D. \& Borisy, G. G. Cellular motility driven by assembly and disassembly of actin filaments. Cell 112, 453-465 (2003).

40. Fromer, M. et al. De novo mutations in schizophrenia implicate synaptic networks. Nature 506, 179-184 (2014).

41. Smoller, J. W. et al. Identification of risk loci with shared effects on five major psychiatric disorders: a genome-wide analysis. Lancet $\mathbf{3 8 1}$ 1371-1379 (2013)

42. Viana, J. et al. Schizophrenia-associated methylomic variation: molecular signatures of disease and polygenic risk burden across multiple brain regions. Hum. Mol. Genet. 26, 210-225 (2017).

43. Rees, E. et al. CNV analysis in a large schizophrenia sample implicates deletions at 16p12.1 and SLC1A1 and duplications at 1p36.33 and CGNL1. Hum. Mol. Genet. 23, 1669-1676 (2014).

44. Sarasua, S. M. et al. Association between deletion size and important phenotypes expands the genomic region of interest in Phelan-McDermid syndrome (22q13 deletion syndrome). J. Med. Genet. 48, 761-766 (2011).

45. Wu, Y., Yao, Y. G. \& Luo, X. J. SZDB: a database for schizophrenia genetic research. Schizophr. Bull. 43, 459-471 (2017).

46. Lanz, T. A. et al. STEP levels are unchanged in pre-frontal cortex and associative striatum in post-mortem human brain samples from subjects with 
schizophrenia, bipolar disorder and major depressive disorder. PLOS. ONE 10, e0121744 (2015).

47. Thorleifsson, G. et al. Genome-wide association yields new sequence variants at seven loci that associate with measures of obesity. Nat. Genet. 41, 18-24 (2009).

48. De, R., Hu, T., Moore, J. H. \& Gilbert-Diamond, D. Characterizing gene-gene interactions in a statistical epistasis network of twelve candidate genes for obesity. BioData Min. 8, 45 (2015).

49. Rzehak, P. et al. DNA-methylation and body composition in preschool children: epigenome-wide-analysis in the European Childhood Obesity Project (CHOP)-Study. Sci. Rep. 7, 14349 (2017).

50. Sugawara, N. et al. Body composition in patients with schizophrenia: comparison with healthy controls. Ann. Gen. Psychiatry 11, 11 (2012).

51. Jurvansuu, J. et al. Transmembrane protein 18 enhances the tropism of neural stem cells for glioma cells. Cancer Res. 68, 4614-4622 (2008).

52. Brennand, $\mathrm{K}$. et al. Phenotypic differences in hiPSC NPCs derived from patients with schizophrenia. Mol. Psychiatry 20, 361-368 (2015).

53. Lin, X. H. et al. Opposite changes in phosphoinositide-specific phospholipase $C$ immunoreactivity in the left prefrontal and superior temporal cortex of patients with chronic schizophrenia. Biol. Psychiatry 46 1665-1671 (1999).
54. Udawela, M. et al. Phospholipase $\mathrm{C}$ beta 1 expression in the dorsolateral prefrontal cortex from patients with schizophrenia at different stages of illness. Aust. N.Z. J. Psychiatry 45, 140-147 (2011).

55. Koh, H. Y. et al. Deficits in social behavior and sensorimotor gating in mice lacking phospholipase C $\beta 1$. Genes. Brain. Behav. 7, 120-128 (2008).

56. Schultz, C. Lipid-induced phenotypes. Nat. Chem. Biol. 2, 396-398 (2006).

57. Zhu, Y. et al. Orexin receptor type-1 couples exclusively to pertussis toxininsensitive G-proteins, while orexin receptor type-2 couples to both pertussis toxin-sensitive and -insensitive G-proteins. J. Pharmacol. Sci. 92, 259-266 (2003).

58. Wockner, L. F. et al. Genome-wide DNA methylation analysis of human brain tissue from schizophrenia patients. TranslationalPsychiatry 4, e339-e339 (2014).

59. Bowen, E. F. W. et al. DLPFC transcriptome defines two molecular subtypes of schizophrenia. Transl. Psychiatry 9, 147 (2019).

60. Boks, M. P. et al. Current status and future prospects for epigenetic psychopharmacology. Epigenetics 7, 20-28 (2012).

61. Martin, M. V., Mirnics, K., Nisenbaum, L. K. \& Vawter, M. P. Olanzapine reversed brain gene expression changes induced by phencyclidine treatment in nonhuman primates. Mol. Neuropsychiatry 1, 82-93 (2015).

62. Pratto, F. et al. Recombination initiation maps of individual human genomes. Science 346, 1256442 (2014) 